\section{Psychosoziale Aspekte der Sucht bei Migranten}

Christian Haasen ${ }^{1}$, Mehmet Ali Toprak², Oktay Yagdiran ${ }^{1}$, Eva Kleinemeier ${ }^{1}$

${ }^{1}$ Universitätsklinikum Hamburg-Eppendorf, Klinik und Poliklinik für Psychiatrie und Psychotherapie (Dir.: Prof. Dr. Dieter Naber), Hamburg

2 KODROBS Suchtberatungsstelle Hamburg-Wilhelmsburg
Zusammenfassung: Bisherige Untersuchungen über die Situation süchtiger Migranten in Deutschland ergeben einen unzureichenden Überblick. Dennoch zeigen einige Daten als auch Erfahrungsberichte, dass unterschiedliche Aspekte der Sucht bei Migranten vorhanden sind, die therapeutische Konsequenzen haben könnten. Daher wurde eine Befragung von süchtigen Migranten unternommen, um spezifische psychosoziale Aspekte zu erfassen. Es wurden 51 süchtige Migranten (davon nur 8 Frauen) in einer Drogenberatungsstelle in Hamburg-Wilhelmsburg zum Suchtverlauf als auch zu migrationsspezifischen Aspekten und Konfliktbereichen befragt. Das Alter bei Beginn des regelmäßigen Konsums lag bei 20 Jahren und die Familienstruktur war bei zwei Dritteln noch intakt. Der Schweregrad der Sucht war bei denjenigen mit Schulabschluss, bei denen mit Berufsausbildung und bei denen mit eher guten Deutschkenntnissen signifikant niedriger. Der Schweregrad der Sucht zeigte einen hochsignifikanten Zusammenhang mit Konfliktbereichen in der Familie und der sozialen Umgebung. Prognostisch günstig erscheinen das höhere Einstiegsalter und die intaktere Familienstruktur. Beides sollte jedoch auch zu einer stärkeren familientherapeutischen Orientierung führen. Ungünstig ist dagegen der starke Zusammenhang mit negativen sozialen Faktoren, da insgesamt Migranten in der deutschen Gesellschaft erschwerten sozialen Bedingungen ausgesetzt sind. Der Zusammenhang zwischen besseren Deutschkenntnissen und einem weniger schweren Suchtverlauf könnte als Hinweis auf die positive Wirkung einer besseren Integration verstanden werden.

Schlüsselwörter: Migration - kulturelle Faktoren - Familie

Psychosocial Aspects of Addiction among Migrants: So far there are insufficient studies available on the situation of addicted migrants. However, some of the data as well as experience reports point out differences with regard to addiction among migrants, which could have therapeutic consequences. Therefore this study on addicted migrants was undertaken to come up with specific psychosocial aspects. 51 addicted migrants (only 8 of them women) were interviewed in an addiction consultation centre in Hamburg-Wilhelmsburg on their course of addiction, migration specific aspects and other areas of conflict. The age at initiation of regular

Suchttherapie 2001; 2: 161-166

(c) Georg Thieme Verlag Stuttgart · New York ISSN 1439-9903 consumption was 20 years and the family structure was still intact for two thirds of them. The severity of addiction was significantly lower for those with a school diploma, as well as for those with vocational training and for those with better German proficiency. The severity of addiction showed a highly significant relationship with conflicts with the family and the surrounding social environment. A good prognostic indicator seems to be the later age of initiation and the intact family structure, which however should lead to a stronger family-therapeutic orientation, especially when considering the high relationship with conflicts with the family. Unfavourable seems to be the high correlation with negative social factors, since migrants are already faced with more difficult social conditions in the German society. The relationship between better German proficiency and a less severe course of addiction can be interpreted as a positive effect of a better integration.

Key words: Migration - Cultural Factors - Family

\section{Einleitung}

Die Forschung auf dem Gebiet der Suchtstörungen sowie deren Behandlung bei Migranten ist in Deutschland noch in den Anfängen, obwohl die Bedeutung für diese Untergruppe schon mehrfach auch bei Suchtfachkonferenzen betont wurde [1]. Es gibt bisher keine bundesweiten Untersuchungen zu Suchterkrankungen bei Migranten. Einige Ergebnisse der transkulturellen Forschung können hilfreich sein, um Unterschiede im Suchtverlauf bei Migranten zu verstehen: Unterschiede im Einstiegsalter (z.B. in der Türkei erst im Alter von Mitte bis Ende 20, nur selten in der Pubertät [2]), in der Geschlechterverteilung (z.B. in Mexiko extrem hohe Abstinenzrate für Frauen [3]) und der Werte und Normen [4] bezüglich der Substanzen.

Einige wenige Studien, aber auch veröffentlichte Erfahrungsberichte über Suchtstörungen unter Migranten zeigen, dass die Anzahl der suchtmittelabhängigen Migranten stark angestiegen ist. Anfang der 80er Jahre wird durch eine Studie über die Versorgung drogenabhängiger „Ausländer“ in Berlin erstmals auf die Quantität und Qualität dieses Problems hingewiesen [5]. In dieser Studie wird festgestellt, dass von 1981 bis 1983 in West-Berlin etwa 1500 drogenabhängige Migranten den Versorgungseinrichtungen bekannt waren. Bei einer Befragung einiger Drogenberatungsstellen in Deutschland stellen Höhn \& Stork [6] ebenfalls fest, dass die Problematik 
des schädlichen Drogenkonsums bei Migranten sowie die Arbeit mit ihnen in diesen Einrichtungen unzureichend thematisiert wird.

Lazaridis [7] kommt bei einer Auswertung der Daten von allen ausländischen Patienten, die von 1975 bis 1982 erstmalig in psychiatrischen Einrichtungen Niedersachsens wegen Suchterkrankungen stationär aufgenommen und behandelt wurden, zu dem Ergebnis, dass von insgesamt 1461 stationär eingewiesenen ausländischen Patienten 228 (15,6\%) wegen Suchterkrankungen behandelt werden. In dieser Studie wird darauf hingewiesen, dass diese Ergebnisse nur die Spitze eines Eisberges darstellen. Die tatsächliche Erkrankungsrate liegt um ein Vielfaches höher.

Die in verschiedenen Städten Deutschlands durchgeführten Untersuchungen weisen auf einen stark zunehmenden Anteil von suchtmittelabhängigen Migranten hin. Zum Beispiel berichtet Philippi [8], dass unter den 1994-1995 erstmals erfassten Drogenabhängigen in Frankfurt ca. 37\% Migranten sind. Ebenfalls wird der Anteil in Essen auf $21 \%$ geschätzt [9]. In dem Bericht von Marinovic [10] wird der Anteil der drogenabhängigen Migranten in der offenen Szene in Stuttgart auf $50 \%$, phasenweise auf $70 \%$ geschätzt.

Nach einem noch nicht veröffentlichten Bericht vom „Projekt Laufwerk“ in Hamburg liegt der Anteil der drogenabhängigen Migranten in der Drogenszene bei 33-35\%. Dagegen liegt in Hamburg der Anteil derjenigen, die ambulante oder stationäre Suchtkrankenhilfe in Anspruch nehmen, bei ca. 5-10\%. Die Situation abhängiger Migranten in Hamburg wurde anhand einer Auswertung von drei Stichproben untersucht [11]. Die 1. Stichprobe umfasste alle Aufnahmen von Migranten in die Klinik für Psychiatrie des Universitätsklinikums Hamburg-Eppendorf in den Jahren 1993 und 1994 mit einer Suchtstörung nach ICD-10 im Rahmen einer Prävalenzstudie zu psychiatrischen Störungen bei Migranten [12]. Die 2 . Stichprobe war eine repräsentative Stichprobe von Heroinabhängigen im niedrigschwelligen Entzug im Klinikum NordOchsenzoll von 1993 bis 1995 [13] unter besonderer Berücksichtigung der Migranten. Die 3. Stichprobe, die sich deutlich von den beiden anderen aufgrund der Erhebungsorte unterschied, untersuchte im Rahmen eines Forschungsprojektes, finanziert vom Bundesministerium für Bildung und Forschung (BMBF), zu psychiatrischen Störungen bei Heroinabhängigen, die Ergebnisse bei denjenigen mit ausländischer Staatsangehörigkeit. Es waren drei Gruppen, die sich stark unterscheiden und dennoch einen Einblick in die Situation der süchtigen Migranten in einer größeren Stadt wie Hamburg zulassen.

Das erste wichtige Ergebnis der Analyse aller drei Stichproben ist die Tatsache, dass der Anteil der Migranten in den Suchteinrichtungen mit ca. 8-10\% unterrepräsentiert ist. Das bedeutet, dass die Bedürfnisse der abhängigen Migranten durch die Drogeneinrichtungen nicht gedeckt werden. Weiterhin wird deutlich, dass abhängige Migranten, die Drogeneinrichtungen in Anspruch nehmen, noch gut sozial und/oder familiär integriert sind. Dieses Ergebnis wird auf eine positive Selektion zurückgeführt. Außerdem wird in dieser Studie festgestellt, dass Migranten eine stärkere Integration in die Familie aufweisen. Dieses wird als eine mögliche Erklärung für die Unterrepräsentanz der Migranten in den Suchthilfe- einrichtungen angesehen. Die bessere familiäre Integration korreliert nicht mit der deutlich schlechteren schulischen und beruflichen Situation.

Bei einer Auswertung der Angaben aller Drogentherapieeinrichtungen in Baden-Württemberg kommt Baudis [14] zu dem Ergebnis, dass die Belegung mit Migranten im Schnitt etwa bei 15-18\% liegt. Dagegen aber liegt der Anteil in der offenen Gruppenarbeit bei 30\%, in der Straßenarbeit sogar bei $50 \%$. In einer in Frankfurt und London durchgeführten Studie von Gaitanides [15] wird festgestellt, dass der Anteil an Migranten in der offenen Szene und bei den niedrigschwelligen Angeboten bei ca. $40 \%$ liegt. Dagegen aber liegt diese Quote bei den Drogenberatungsstellen mit 10-15\% sehr viel niedriger. In den stationären Therapieeinrichtungen und betreuten Wohngemeinschaften ist dieser Anteil, mit Ausnahme der zielgruppenorientiert arbeitenden Therapieeinrichtungen, noch geringer. Hier wird von einer Einrichtung berichtet, die ihren Migrantenanteil durch den Einsatz einer türkischen und einer kroatischen Beratungskraft von $8 \%$ (1988) auf 31\% (1995) erhöhte. Ähnlich hoch lag in London die Differenz zwischen Einrichtungen mit und ohne afrikanische Mitarbeiter.

Von den Niederlanden berichtet Trautmann [16], dass sich alle Migrantengruppen mit Ausnahme von Spaniern mit Anpassungs- und Integrationsproblemen konfrontiert sehen. Dies lässt sich unter anderem an der Tatsache ablesen, dass sie im Hinblick auf einen problematischen Drogenkonsum überdurchschnittlich gefährdet sind. Bei einer Auswertung der im landesweiten Alkohol- und Drogen-Informationssystem (LADIS) über die Klientenpopulation der niederländischen Drogenhilfeeinrichtungen gesammelten Daten wird festgestellt, dass bei einem Migrantenanteil von 6\% an der Gesamtbevölkerung (15,6 Millionen) der Anteil von Migranten mit Suchtstörungen bei $28 \%$ liegt. Weiterhin wird festgestellt, dass etwa $66 \%$ der Migranten gegenüber $52 \%$ niederländischer Herkunft ein Behandlungsprogramm vorzeitig verlassen. Dieser Umstand veranlasste die Politik, für die Praxis neue Wege und Strategien in Beratung und Therapie der Suchtstörungen bei Migranten zu suchen.

Eine besondere Untergruppe der Migranten stellen die Flüchtlinge dar. Insgesamt wird bei Flüchtlingen aufgrund der zum Teil extremen psychosozialen Belastungen ein höheres Risiko für süchtiges Verhalten beschrieben [17-19]. Dennoch wurden auch in dieser Untergruppe bestimmte protektive Faktoren eruiert, die möglicherweise auch für andere Migrantengruppen zutreffen: So wurde das Leben in einer ethnisch-ähnlichen Gemeinschaft in der fremden Gesellschaft als protektiv beschrieben [20] als auch ein starker religiöser Glaube [21], eine ideologische Weltanschauung [18] und eine ausgedehnte Familie [21]. Dennoch können auch diese zunächst protektiven Faktoren zu Integrationsschwierigkeiten führen, die wiederum neue Risiken darstellen.

\section{Material und Methoden}

Obwohl die wenigen Ergebnisse aus der Literatur deutlich machen, dass die Migration einen Entwicklungsbruch mit einer resultierenden höheren Suchtrate darstellt, fehlen bislang Untersuchungen, die spezifische psychosoziale Faktoren bei dieser Entwicklung herausarbeiten. Die vorliegende Arbeit 
stellt eine Untersuchung über eine Stichprobe von Migranten vor, die im Zeitraum von Januar bis April 1998 die Suchtberatungsstelle KODROBS Wilhelmsburg/Hamburg des Trägers Jugend hilft Jugend e.V. um Hilfe aufgesucht haben. Die Suchtberatungsstelle liegt in Wilhelmsburg, einem Stadtteil von Hamburg mit einem Migrantenanteil von 33\%. Die überwiegende Mehrheit davon sind Migranten türkischer Herkunft (52\% in diesem Stadtteil). Der Anteil der Migranten in Hamburg beträgt dagegen zirca $16 \%$.

Die Beratungsstelle zeichnet sich durch einen Suchtmittelübergreifenden Ansatz aus und hat einen Schwerpunkt in der Arbeit mit abhängigen Migranten. Bestandteil des ausstiegsorientierten Konzepts der Beratungsstelle sind Suchtberatung, Therapievorbereitung, Suchtbegleitung, psychosoziale Betreuung, Suchtakupunktur und ambulante Suchttherapie. Die Erfahrung in der Arbeit mit suchtmittelabhängigen Migranten führte zur Entwicklung eines spezifischen ambulanten Suchttherapiekonzepts für diese Gruppe.

Es wurden 51 suchtmittelabhängige Migranten (alle konsekutiven Migranten, die zu einem Beratungsgespräch kamen) mit Hilfe eines selbst entwickelten Fragebogens befragt. Folgende Personen wurden im Rahmen dieser Arbeit als Migranten definiert: Personen, die in einem anderen Land als Deutschland geboren wurden, anschließend jedoch, unabhängig von ihrem Alter, dorthin migriert sind. Es wurden ebenfalls Migranten mit berücksichtigt, die inzwischen im Besitz der deutschen Staatsangehörigkeit waren, der Geburtsort jedoch im Ausland war.

Der erste Teil des Fragebogens befasst sich mit soziodemografischen Daten, wie Alter, Geschlecht, Familienstand, Wohnund Arbeitssituation, als auch mit migrationsspezifischen Daten, wie Aufenthaltsstatus, Sprache, Alter bei Migration. Weiterhin wurden der Suchtverlauf, das Konsumverhalten und der Schweregrad des Suchtmittelkonsums (analog dem EuropASI [22]) erfasst. Im zweiten Teil des Fragebogens wurden drei mögliche Konfliktbereiche evaluiert: der Bereich der Diskriminierungserfahrungen, transgenerationale Konfliktbereiche, mögliche Partnerkonflikte und zum Schluss eine Bewertung des Schweregrades dieser drei Konfliktbereiche (ebenfalls analog des EuropASI) zusammen.

\section{Ergebnisse}

\section{Soziodemografische Daten}

Die wichtigsten soziodemografischen Daten werden in Tab. 1 aufgeführt. 8 der 51 Befragten waren Frauen. Das durchschnittliche Alter bei der Befragung lag bei 26,7 Jahren $(\mathrm{SD}=6,7$; Min. =14; Max. =43). Männer waren signifikant älter als Frauen $(t=3,74 ; p<0,001)$, tendenziell eher verheiratet $\left(\chi^{2}=5,18 ; p<0,1\right)$ und hatten häufiger mindestens ein Kind $\left(\chi^{2}=7,2 ; p<0,01\right)$. Die Befragten ohne Kinder waren mit 23,6 Jahren signifikant jünger als diejenigen mit Kindern (30,9 Jahre ; $\mathrm{t}=4,6 ; \mathrm{p}<0,001)$.

35 (69\%) der Befragten befanden sich in einer Partnerschaft, Frauen signifikant seltener $\left(\chi^{2}=8,4 ; \mathrm{p}<0,01\right)$. Diejenigen mit PartnerIn waren mit 28,0 Jahren signifikant älter als diejenigen ohne PartnerIn (24,0 Jahre; $t=2,0 ; p<0,05)$. Von den Befragten wohnten 10 (19,6\%) alleine, 36 (70,6\%) mit der
Tab. 1 Geschlechtsspezifische soziodemografische Daten

\begin{tabular}{llc}
\hline & männlich & weiblich \\
\hline Anzahl & & \\
Alter & 43 & 8 \\
Familienstand & 28,1 & 19,5 \\
feste Partnerschaft & $65 \%$ ledig & $100 \%$ ledig \\
Anteil mit Kindern & $77 \%$ & $25 \%$ \\
Anteil arbeitslos & $51 \%$ & $0 \%$ \\
Anteil mit Schulabschluss & $72 \%$ & $100 \%$ \\
Anteil mit Berufsausbildung & $74 \%$ & $62 \%$ \\
& $35 \%$ & $13 \%$
\end{tabular}

Familie, 3 (5,9\%) mit anderen und 2 (3,9\%) waren ohne festen Wohnsitz. Zur Zeit der Befragung waren 39 (76\%) arbeitslos, $2(4 \%)$ in Teilzeit, $4(8 \%)$ in Vollzeit und $6(12 \%)$ gelegentlich beschäftigt. $37(72,6 \%)$ der Befragten hatten einen Schulabschluss und $14(27,4 \%)$ hatten keinen Schulabschluss. Zum Zeitpunkt der Befragung hatten 16 (31,4\%) eine Berufsausbildung abgeschlossen. Bei der Wohnsituation, beruflichen Situation und bezüglich der Ausbildung waren keine geschlechtsspezifischen Unterschiede zu verzeichnen.

\section{Migrationsspezifische Daten}

Von den Befragten stammten 34 Personen (66,0\%) aus der Türkei und 17 Personen (34,0\%) aus folgenden Ländern: 1 aus Bosnien (2,0\%), 1 aus Kroatien (2,0\%), 4 aus dem Iran (8,0\%), 2 aus Italien (4,0\%), 2 aus Spanien (4,0\%), 7 aus Portugal (14,0\%). 18 Personen (35,3\%) kamen aus einer Großstadt, 18 (35,3\%) aus einer Kleinstadt und 15 (29,4\%) aus dörflichländlichen Gebieten.

Zum Zeitpunkt der Migration nach Deutschland lag das Alter durchschnittlich bei 9,3 Jahren ( $S D=8,1)$, bei denjenigen aus der Türkei bei 6,5 Jahren und bei den anderen bei 15,0 Jahren $(\mathrm{t}=4,0 ; \mathrm{p}<0,1)$. Von den Befragten hatten 43 Personen $(86 \%)$ eine unbefristete, 4 Personen ( $8 \%$ ) eine befristete Aufenthaltserlaubnis, 3 Personen (6\%) hatten Asyl erhalten und einer hatte die deutsche Staatsangehörigkeit.

Bezüglich der Deutschkenntnisse zeigte sich folgendes Ergebnis: 8 Personen $(15,7 \%)$ sprachen fließend Deutsch, 22 Personen (43,0\%) gut, 14 Personen (27,5\%) mäßig und 7 Personen $(13,8 \%)$ schlecht Deutsch. Somit sprachen 58,8\% fließend bis gut und $41,2 \%$ mäßig bis schlecht. Hier zeigte sich ein geschlechtsspezifisch tendenzieller Unterschied $\left(\chi^{2}=3,2\right.$; $\mathrm{p}<0,1)$ - Frauen sprachen besser Deutsch als Männer.

\section{Daten zur Suchtentwicklung und Suchtverlauf}

Hinsichtlich der Suchtmittel konsumierten zum Zeitpunkt der Befragung 49 Personen (96,0\%) als Hauptdroge Heroin, einer Alkohol und eine Person Cannabis. Alle Befragten waren Konsumenten weiterer Suchtmittel, darunter Alkohol, Cannabis, Kokain und Benzodiazepine. Von den 8 Frauen konsumierten 7 primär Heroin, eine Cannabis.

Das Durchschnittsalter beim ersten Kontakt mit Drogen lag bei 17,0 Jahren ( $\mathrm{SD}=3,2$; Min. = 12; Max. = 32) ohne geschlechtsspezifische Unterschiede $(t=1,5 ; n$.s.). Das Durchschnittsalter bei Beginn des regelmäßigen Konsums von Suchtmitteln lag 


\begin{tabular}{|c|c|c|}
\hline & $\begin{array}{l}\text { Schweregrad Sucht } \\
\text { Skala 0-9: } \mathrm{M}=7,8\end{array}$ & $\begin{array}{l}\text { Schweregrad Konfliktbereiche } \\
\text { Skala 0-9: } \mathrm{M}=7,0\end{array}$ \\
\hline Alter & $r=0,16 ;$ n.s. & $r=0,23 ; p<0,05$ \\
\hline Geschlecht & $\mathrm{t}=0,9 ; \mathrm{n} . \mathrm{s}$ & $\mathrm{t}=0,4 ; \mathrm{n} . \mathrm{s}$ \\
\hline türkisch/andere Herkunft & $\mathrm{t}=1,6 ; \mathrm{n} . \mathrm{s}$ & $\mathrm{t}=1,5 ; \mathrm{n} . \mathrm{s}$ \\
\hline Berufsausbildung & $t=-2,1 ; p<0,05$ & $t=-2,1 ; p<0,05$ \\
\hline Schulabschluss & $t=-2,2 ; p<0,05$ & $t=-1,8 ; p<0,05$ \\
\hline Deutschkenntnisse & $t=-2,0 ; p<0,05$ & $\mathrm{t}=1,3 ;$ n.s. \\
\hline Erstalter Drogenkonsum & $r=-0,33 ; p<0,05$ & $r=-0,18 ;$ n.s. \\
\hline $\begin{array}{l}\text { Alter bei Beginn eines } \\
\text { regelmäßigen Konsums }\end{array}$ & $r=-0,19 ;$ n.s. & $r=-0,11 ; n . s$. \\
\hline Alter erster Kontakt Suchthilfe & $r=-0,08 ;$ n.s. & $r=-0,16 ;$ n.s. \\
\hline stationäre Therapieerfahrung & $t=3,5 ; p<0,01$ & $\mathrm{t}=1,9 ;$ n.s. \\
\hline ambulante Therapieerfahrung & $\mathrm{t}=0,4 ;$ n.s. & $\mathrm{t}=0,9 ; \mathrm{n} . \mathrm{s}$ \\
\hline Schweregrad Konfliktbereiche & $r=0,67 ; p<0,001$ & - \\
\hline
\end{tabular}

Tab. 2 Zusammenhang der soziodemografischen und Krankheitsfaktoren mit dem Schweregrad der Sucht und Konfliktbereiche bei 20 Jahren ( $S D=4,5$; Min.=13; Max.=35). Das Durchschnittsalter bei Männern lag bei 20,6 und bei Frauen bei 16,4 $(\mathrm{t}=2,7 ; \mathrm{p}<0,1)$. Das Durchschnittsalter beim ersten Kontakt mit dem Suchthilfesystem lag bei 25,8 Jahren ( $S D=6,0$; Min. =14,0; Max. =43,0); Männer waren dabei mit 27 Jahren signifikant älter als Frauen mit 19 Jahren $(t=3,7 ; p<0,001)$. 7 Personen $(13,8 \%)$ hatten stationäre und 8 Personen $(16,0 \%)$ ambulante Therapieerfahrung.

Hinsichtlich des Schweregrades der Sucht lag der Durchschnittswert bei 7,8 auf einer Skala von 0 (kein echtes Problem) bis 9 (extremes Problem). Die wichtigsten Zusammenhänge mit soziodemografischen Faktoren und Krankheitsvariablen werden in Tab. 2 angegeben.

\section{Daten zu Konfliktbereichen}

Die Angaben der Probanden über Probleme mit der sozialen Umgebung, vor allem Diskriminierungserfahrungen, wurden auf einer Skala von 0 (gar nicht) bis 4 (extrem) beurteilt. Der Durchschnittswert bei Problemen mit der sozialen Umgebung lag bei 2,2. Ebenfalls wurden die Konflikte mit den Eltern auf einer Skala von 0 (gar nicht) bis 4 (extrem) beurteilt. Hier lag der Durchschnitt bei 3,0. Außerdem wurden die Konflikte mit Partnern auf einer Skala von 0 (gar nicht) bis 4 (extrem) bewertet. Hier lag der Durchschnittswert bei 2,5. Zuletzt wurden die drei Konfliktbereiche - mit den Eltern, mit Partnern und mit der sozialen Umgebung - auf einer gemeinsamen Skala von 0 (kein Problem) bis 9 (extremes Problem) beurteilt. Hier lag der durchschnittliche Schweregrad bei 7,0. Die wichtigsten Zusammenhänge mit soziodemografischen Daten und Krankheitsvariablen werden in Tab. $\mathbf{2}$ angegeben. Insgesamt zeigten sich keine signifikanten geschlechtsspezifischen Unterschiede bei den Konfliktbereichen.

Der Schweregrad der Sucht zeigte eine hochsignifikante Korrelation mit der Beurteilung der Probleme in der sozialen Umgebung $(r=0,5 ; p<0,001)$, eine hochsignifikante Korrelation mit der Beurteilung der Probleme mit den Eltern $(r=0,5$; $p<0,001$ ), jedoch keine Korrelation mit Partnerschaftsproblemen $(r=0,12$; n.s.). Der Schweregrad der drei Konfliktbereiche zeigte eine hochsignifikante Korrelation mit dem Schweregrad der Sucht (Tab. 2).

\section{Diskussion}

Die vorliegenden Ergebnisse können als repräsentativ für die ambulante Betreuung von suchtmittelabhängigen Migranten in Hamburg angesehen werden, da die Untersuchung in einer ambulanten Einrichtung stattfand, in der der Migrantenanteil 25 bis 30\% beträgt, während in anderen vergleichbaren Einrichtungen der Anteil bei etwa 5-10\% liegt. Dieses liegt daran, dass in dieser Einrichtung ein Mitarbeiter nicht deutscher Herkunft tätig ist und sie in einem Stadtteil liegt, in dem um die 33\% Migranten wohnen. Dennoch erlaubt die begrenzte Größe der Stichprobe nur eingeschränkte Aussagen zu Untergruppen und muss deshalb eher als hypothesengenerierend betrachtet werden.

Zusammenfassend zeigen die Ergebnisse einen hohen Anteil suchtmittelabhängiger Männer, die zu einem Drittel in einer Beziehung leben und mindestens ein Kind haben. Weiterhin leben suchtmittelabhängige Migranten meistens noch bei ihren Familien. Drei Viertel haben einen Schulabschluss, nur ein Drittel hat eine abgeschlossene Berufsausbildung. Bei den migrationsspezifischen Daten fällt vor allem das junge Alter zum Zeitpunkt der Migration als auch der Zusammenhang zwischen dem Schweregrad der Sucht und den Deutschkenntnissen auf.

Der hohe Anteil suchtmittelabhängiger Männer deckt sich mit Ergebnissen aus anderen Untersuchungen. Zwei mögliche Erklärungen dafür könnten sein, dass:

a) Frauen durch die kulturell bedingten festen Rollen in der Familie andere Probleme und Konfliktbewältigungsmechanismen entwickeln als Männer aus gleichen Familien. Die geschlechtsspezifische Erwartungshaltung des sozialen Umfeldes, insbesondere der Eltern, könnte bei den Geschlechtern die körperlich emotionalen Reaktionen auf psychosoziale Belastungen in der Art und Weise bestimmen, dass Männer eher suchtmittelabhängig werden als Frauen,

b) suchtmittelabhängige Frauen die Hilfe der Einrichtungen im Suchtbereich nicht in Anspruch nehmen. Es bedarf epidemiologischer Untersuchungen, um diesen Zusammenhang klären zu können.

Ein prognostisch wichtiges Ergebnis dieser Untersuchung, welches sich mit Ergebnissen aus der Literatur [20,21] deckt, ist, dass ein Drittel der suchtmittelabhängigen Migranten, 
mehrheitlich männlichen Geschlechts in einer Partnerschaft bzw. in einer Ehe lebt, mit mindestens einem Kind. Der Grund mag darin liegen, dass Migranten durch die starke Bindung zur Herkunftsfamilie, die auf die Heirat von Kindern enormen Einfluss ausüben, im jüngeren Alter heiraten als vergleichsweise Deutsche. Die Einflussnahme der Eltern auf die Heirat der Kinder ist aus deren Sicht dadurch zu erklären, dass die Familiengründung zu einer Verstärkung individuell-sozialer Verantwortung führt, so dass dadurch möglicherweise vorhandene Störungen wie Sucht „gelöst“ werden. Die Ergebnisse zu Geschlecht und Partnerschaft sprechen für eine differenzierte Suchtbehandlung: Bei Männern müsste stärker familientherapeutisch gearbeitet werden, während bei Frauen der Zusammenhang zwischen fehlender partnerschaftlicher Struktur und der Suchtentwicklung therapeutisch herausgearbeitet werden sollte.

In Bezug auf die Wohnsituation von Migranten mit Suchtproblemen lässt sich feststellen, dass die überwiegende Mehrheit drogenabhängiger Migranten bei ihren Familien lebt. Sie sind sozial und/oder familiär gut integriert. Suchtmittelabhängige Migranten werden von ihren Angehörigen ohne Vorbehalt unterstützt: Die familiären Bindungen sind so stark, dass die Angehörigen es nicht wagen würden, sie auf die Straße zu setzen, was hierzulande eine allgemein gültige Beratungspraxis darstellt. Das bedeutet, dass Suchthilfeeinrichtungen bei Beratung und Behandlung von Migranten auf dieses spezifische Beziehungsgeflecht respektvoll und behutsam eingehen müssen und spezifische Konzepte erarbeitet werden sollten. Eine in familientherapeutischen Settings durchgeführte Behandlung würde der Situation und den Bedürfnissen der Migranten gerechter werden als andere traditionelle Suchtbehandlungsformen.

Als wichtiger sozialer Aspekt erscheint das Verhältnis derjenigen mit Schulabschluss und derjenigen mit abgeschlossener Berufsausbildung: Obwohl drei Viertel einen Schulabschluss haben, hat nur ein Drittel eine abgeschlossene Berufsausbildung. Hier potenzieren sich die eher schon schlechten Berufsperspektiven junger Menschen bei dieser Untergruppe von Migranten.

Bei den migrationsspezifischen Daten fällt vor allem das Alter zum Zeitpunkt der Migration auf, welche durchschnittlich in einer sehr vulnerablen Phase der kindlichen Entwicklung stattfindet. In den Gesprächen mit den Betroffenen wurde $\mathrm{zu}$ diesem Bereich neben den erfragten Daten von vielen angemerkt, dass sie außerdem mehrfach wieder zurück in die Heimat geschickt wurden. Sie wurden also hin- und hergeschoben und hatten keine festen Bezugspersonen, was ein Gefühl von „Verlassenwerden“, „Unerwünschtsein“ oder „Nichtgeliebtsein“ auslöste. Sie reagierten auf die Migration mit Angst, mit Rückzug bis hin zu Isolierung, mit Trauer, mit Unsicherheit, mit Sprachlosigkeit in der Familie und mit Sprachschwierigkeiten. Hierzu passt außerdem die trotz häufig abgeschlossener Schulbildung in Deutschland dennoch hohe Rate derjenigen mit eher schlechten Deutschkenntnissen.

Bei der Betrachtung der Suchtentwicklung fällt auf, dass die wichtigsten zeitlichen Eckpunkte (erster Kontakt mit Drogen, Beginn des regelmäßigen Konsums, erster Kontakt mit dem Suchthilfesystem) später liegen als bei Deutschen. Dieses im Einklang mit der transkulturellen Literatur [2] stehende
Ergebnis ist einerseits prognostisch eher günstig zu bewerten Andererseits sprechen die Zeitpunkte für einen stärkeren Zusammenhang mit gesellschaftlichen Anpassungsschwierigkeiten: Der Suchtbeginn findet zu einem Zeitpunkt statt, wo Migranten sich beruflich und familiär orientieren müssen, aber auch die ersten enttäuschten Hoffnungen und Diskriminierungserfahrungen durchgemacht haben. Dieses wird dadurch bestätigt, dass der Schweregrad der Sucht bei denen mit Berufsausbildung, mit Schulabschluss und eher guten Deutschkenntnissen geringer ist als bei den anderen.

Wichtig für therapeutische Überlegungen ist die Tatsache, dass stationäre Therapieerfahrung mit einem geringeren Schweregrad der Sucht zusammenhängt, während ambulante Therapieerfahrung keinen Zusammenhang mit dem Schweregrad der Sucht zeigt. Dieses mag mit der intensiven Auseinandersetzung während einer stationären Therapie zusammenhängen, welches bei heutigen gesundheitspolitischen Überlegungen zur Abschaffung sämtlicher stationärer Suchtbehandlungen mit berücksichtigt werden sollte. Dennoch darf bei dieser Stichprobe das Ergebnis nur vorsichtig gedeutet werden, da nur wenige Migranten Therapieerfahrungen hatten. Dieser Punkt müsste mit größeren Stichproben weiter untersucht werden.

Die Konfliktbereiche bestätigen einerseits die Bedeutung von Konflikten mit Eltern und der sozialen Umgebung; diese sind vermutlich nicht sehr viel anders als bei denjenigen deutscher Herkunft. Wichtig erscheint das Ergebnis, dass Konflikte in der Partnerschaft nicht mit dem Schweregrad der Sucht korrelierten, welches eine wichtige familientherapeutische Bedeutung hat. Andererseits kommt bei süchtigen Migranten ein wichtiger Konfliktbereich hinzu - die Diskriminierungserfahrung, die es bei denen deutscher Herkunft nicht gibt. Von großer Bedeutung ist ebenfalls die Tatsache, dass bessere Deutschkenntnisse mit einem geringeren Schweregrad der Sucht korrelieren, was die Bedeutung einer Integration in die deutsche Gesellschaft unterstreicht. Der Schweregrad aller Konfliktbereiche zeigt einen sehr starken Zusammenhang mit dem Schweregrad der Sucht, welches die Notwendigkeit der spezifischen therapeutischen Maßnahmen unterstreicht. $\mathrm{Zu}-$ sammenfassend bestätigen somit die Ergebnisse die Hypothese, dass der Schweregrad der Sucht mit schlechteren und migrationsspezifischen Lebensbedingungen zusammenhängt, aber die größtenteils intakte Familienstruktur als auch das höhere Alter bei der Suchtentwicklung prognostisch günstige Ansatzpunkte im therapeutischen Prozess bieten.

\section{Literatur}

${ }^{1}$ Loosen W. Fachkonferenz „Sucht 1997“ in Hamburg: Viele Aussiedler werden in Deutschland süchtig. Deutsches Ärzteblatt 1997; 94: 2317

2 Evlice YE. Heroinabhängigkeit in der Türkei. Vortrag III. DeutschTürkischer Psychiatriekongress. Berlin, 1998

${ }^{3}$ Caetano R. Alcohol use among Hispanic groups in the United States. Am J Drug Alcohol Abuse 1988; 14: 293-308

${ }^{4}$ Pfeiffer WM. Suchtbehandlung aus transkultureller Perspektive. In: Krausz M, Haasen C (Hrsg). Langzeitperspektive Süchtigen Verhaltens. Freiburg: Lambertus, 1996

${ }^{5}$ Akbiyik O. Drogenarbeit mit Ausländern. In: Heckmann W (Hrsg). Drogentherapie in der Praxis. Ein Arbeitsbuch für die 90er Jahre. Weinheim, Basel: Beltz, 1991: 156-171 
${ }^{6}$ Höhn R, Stork C. Drogenproblematik junger Ausländer. Informationsdienst zur Ausländerarbeit, 1990; 3: 46-48

${ }^{7}$ Lazaridis K. Epidemiologische Aspekte der Suchterkrankungen bei Ausländern. Hospitalisationsinzidenz in Niedersachsen. Suchtgefahren 1988; 34: 396-400

${ }^{8}$ Philippi R. Drogenkriminalität und Gefährdungspotential. In: Kommunale Ausländerinnen- und Ausländervertretung (KAV) der Stadt Frankfurt a.M (Hrsg). Drogen und Migration: Dokumentation der Anhörung am 4. März 1996 zur Situation Drogenabhängiger und -gefährdeter junger Menschen ausländischer Herkunft in Frankfurt a.M. Bonn, 1996; 67

${ }^{9}$ Goldmann W. Erschreckend hoch. In: Psychosozialer Arbeitskreis Migration im Projekt Gesunde Stadt Essen (Hrsg). Drogenkonsum - Fakten, Hintergründe, Prävention, Ausblicke 3. Veranstaltung der Reihe Migration und Justiz. Essen, 1996; 7

${ }^{10}$ Marinovic P. Streetwork in der offenen Drogenszene mit nichtdeutschen KonsummentInnen. In: Landesstelle gegen die Suchtgefahren in Baden-Württemberg (Hrsg). Sucht und Migration, 1997: 17-20

${ }^{11}$ Haasen C. Migration und Sucht: Empirische Ergebnisse und Zukunftsperspektive. In: Schäfer L, Schmalfuß ES (Hrsg). Euro TC 1996 Strasburg: Europe unlimited! Drugs unlimited? Deutsche Ausgabe: Föderation der Drogenhilfen in Europa e.V. 1997

${ }^{12}$ Haasen C, Lambert M, Yagdiran O, Krausz M. Psychiatric disorders among migrants in Germany: prevalence in a psychiatric clinic and implications for services and research. Eur Psychiatry 1997; 12: 305-310

13 Degkwitz P, Krausz M. Epidemiologie und Dokumentation im Drogenentzug. In: Behrendt K, Degkwitz P, Trüg E (Hrsg). Schnittstelle Drogenentzug. Freiburg: Lambertus, 1995

${ }^{14}$ Baudis R. Gastarbeiterkinder In: Landesstelle gegen die Suchtgefahren in Baden-Württemberg (Hrsg). Sucht und Migration, 1997: 54-64

${ }^{15}$ Gaitanides S. Zugangsbarrieren von Migranten zu den Drogendiensten. In: Deutsche Hauptstelle gegen die Suchtgefahren (Hrsg). Sucht in unserer multikulturellen Gesellschaft. Freiburg i.B: Lambertus, 1998

${ }^{16}$ Trautmann F. Drogenarbeit mit Migranten in den Niederlanden. In: Deutsche Hauptstelle gegen die Suchtgefahren (Hrsg). Sucht in unserer multikulturellen Gesellschaft. Freiburg i. B: Lambertus, 1998: 234-247

${ }^{17}$ Desjarlais R, Eisenberg L, Good B, Kleinman A. World Mental Health: Problems and Priorities in Low-Income Countries. New York: Oxford University Press, 1995

18 Reynell J. Political Pawns: Refugees on the Thai-Kampuchean Border. Oxford: Refugee Studies Programme, 1989

${ }^{19}$ Westermeyer J. Psychiatric services for refugee children. In: Ahearn FL, Athey JL (Hrsg). Refugee children: Theory, Research, and Services. Baltimore MD: The Johns Hopkins University Press, 1991: 127-162

${ }^{20}$ Murphy HBM. Migration, culture, and mental health. Psychol Med 1977; 7: 677-684

${ }^{21}$ McCallin M, Fozard S. The Impact of Traumatic Events on the Psychological Well-Being of Mozambican Refugee Women and Children. Genf: International Catholic Child Bureau, 1990

${ }^{22}$ Kokkevi A, Hartgers C. EuropASI: European adaptation of a multidimensional assessment instrument for drug and alcohol dependence. European Addiction Research 1995; 1: 208-210

\section{Dr. Christian Haasen}

Universitätsklinikum Hamburg-Eppendorf

Klinik und Poliklinik für Psychiatrie und Psychotherapie

Martinistraße 52

20246 Hamburg

E-mail: haasen@uke.uni-hamburg.de 